\title{
Material strength safety factors for the seismic safety assessment of existing RC buildings
}

\author{
Nuno Pereira, Xavier Romão* \\ CONSTRUCT-LESE, Faculty of Engineering, University of Porto, Portugal
}

\section{H I G H L I G H T S}

- Safety factors are proposed to characterize material strength in existing buildings.

- The uncertainty due to the number of tests and strength variability are considered.

- The safety factors are compatible with EC8/3 seismic safety assessment methods.

- Safety factors and survey plans are defined for concrete compressive strength.

- Safety factors and survey plans are defined for reinforcing steel yield strength.

\section{A R T I C L E I N F O}

\section{Article history:}

Received 21 September 2015

Received in revised form 2 May 2016

Accepted 4 May 2016

\section{Keywords:}

Material safety factor

Confidence factor

Material strength estimation

Seismic safety assessment

Existing structure

RC building

\begin{abstract}
A B S T R A C T
New material safety factors $\left(\mathrm{CF}_{\text {mat }}\right)$ are proposed to characterize material strength in existing buildings. These safety factors are developed in order to be compatible with seismic safety assessment procedures defined by current standards such as Eurocode 8 Part 3 . The general theory behind the development of the $\mathrm{CF}_{\text {mat }}$ safety factors considers the uncertainty associated to the number of surveyed structural elements and the inherent variability of the material strength under analysis. The $\mathrm{CF}_{\text {mat }}$ safety factors are developed using a finite population approach where the material properties in a building are discretized by considering one value per element. The proposed theory is used to define specific $\mathrm{CF}_{\text {mat }}$ values and survey plans for the concrete compressive strength and for the reinforcing steel yield strength.
\end{abstract}

(c) 2016 Elsevier Ltd. All rights reserved.

\section{Introduction}

Assessing the seismic performance of existing structures is a matter of high priority in earthquake prone areas. As recognized by earthquake engineering experts and public authorities, evaluating the safety of existing buildings and infrastructures is fundamental. Therefore, specific code-based methods must be developed to address these issues and an adequate calibration of these methods must be carried out to analyse their ability to be used in practice. As such, several standards (e.g. [1-6]) have been recently developed to address the specifics of the seismic safety assessment of existing structures and studies analysing some of their procedures have started to appear [7-11].

One important issue that affects the evaluation of the seismic performance of existing buildings is related to the definition of

\footnotetext{
* Corresponding author.

E-mail address: xnr@fe.up.pt (X. Romão).
}

their material properties, since the original construction quality levels and design standards may be very different from those currently in use. Characterizing these material properties can be achieved in different ways which may lead to different levels of knowledge, depending on the level of detail provided by the survey plans and on the availability and reliability of information about the design. Therefore, the reliability of the structural properties considered in the seismic safety assessment will depend on the correlation between the amount of knowledge gathered about the structure and the confidence about that data. Still, to account for the existing uncertainty, the structural properties need to be defined with values that are on the "safe side".

The current European standard for the seismic safety assessment of existing buildings is the Eurocode 8-Part 3 (EC8/3) [1]. This standard specifies explicit rules regarding the assessment of structural properties in existing buildings, namely regarding the geometry, the structural details and the material properties. Survey plans are specified for all these components in order to conform 
to qualitative knowledge levels (KLs). Associated to each KL, EC8/3 defines a coefficient termed confidence factor (CF) that factors the mean material strength values in order to establish values that are on the "safe side" and to reduce the admissible capacity of the structural elements due to the uncertainty. The connection between the KLs and the CF values has been criticized (e.g. see $[7,8]$ ) due to the lack of objectivity behind the CF values. By only affecting the mean material properties, the $\mathrm{CF}$ does not reflect explicitly the remaining uncertainties, a fact that led to alternative interpretations of this parameter that consider the $\mathrm{CF}$ to be a factor only able to represent the uncertainty about the material properties. Rota et al. [9] modified the CF concept proposed by the EC8/3 and also by the Italian standard NTC-08 [5] and defined a coefficient accounting only for the uncertainty in the material properties. The framework they developed assumed that a multiple uncertainty approach would be more adequate than the methodology proposed by the standards. Monti and Alessandri [10] and Romão et al. [11] presented two generic methods that provide a probability-based approach to calibrate a coefficient $\mathrm{CF}_{\text {mat }}$ accounting for the uncertainty in the material properties. These generic methods formulate coefficients that depend on the statistical analysis of a given number of tests that are performed in the structure to assess the material properties.

The present study follows the fundamental concepts adopted in [11] to derive an alternative safety factor $\mathrm{CF}_{\text {mat }}$ for the mean value of a material strength in existing reinforced concrete (RC) buildings. The fact that the approach in [11] does not include explicitly the sampling uncertainty and material strength disaggregation will be addressed by the methodology proposed herein. This approach will introduce an adaptive probability-based formulation defining a set of sampling plans and $\mathrm{CF}_{\text {mat }}$ values (similar to the concepts of KLs proposed in EC8/3) based on finite population statistics. A comparison will be also made with the original $\mathrm{CF}$ values proposed by $\mathrm{EC} 8 / 3$ in order to check the maximum variability level of the material properties (represented by the coefficient of variation, $\mathrm{CoV}$ ) that is compatible with the approach presented in the code. Furthermore, a survey framework will be presented that includes the definition of different $\mathrm{CF}_{\text {mat }}$ values for the concrete compressive strength and for the reinforcing steel yield strength and that specifies the different number of tests that have to be performed to characterize these material properties.

\section{Brief review of current standard-based methods to assess material properties in existing buildings}

Standards for the seismic safety assessment of existing RC buildings establish that a given number of tests must be carried out in a structure to determine the material properties, namely to characterize the concrete compressive strength and the yield strength of the reinforcement. According to these standards, material properties can be characterized by performing destructive tests on a number of material samples extracted from the structural members. Due to the destructive nature of this approach and the costs that it may involve (both direct and indirect), standards also suggest the use of non-destructive tests (NDTs) to complement the data obtained from destructive testing. Still, no specific rules on how to include these auxiliary results are defined. To provide additional details regarding the context of the present study, the procedures proposed by some of these standards are briefly reviewed in the following.

\subsection{Eurocode 8 - Part 3}

EC8/3 defines the minimum number of material samples that must be tested by defining, for each storey and each type of member, the number of tests that guarantees a certain KL. EC8/3 establishes three KLs: KL1, KL2 and KL3, which are termed Limited, Comprehensive and Full, respectively. For each KL, EC8/3 assigns a $\mathrm{CF}$ that will act as a safety factor for the mean value of the material properties accounting for the uncertainty induced by the material sampling plan. The values of the CFs proposed by EC8/3 are 1.35 , 1.20 and 1.00 for KL1, KL2 and KL3, respectively. No distinction is made in the code between the concrete compressive strength and the reinforcing steel yield strength regarding the number of tests that need to be performed and the CF values that are adopted for these two properties. For KL3, three concrete cores and three samples of reinforcing steel bars from each storey and from each type of element must be tested. The number of samples that must be tested is reduced to two and to one for KL2 and KL1, respectively.

\subsection{Italian standard NTC-08}

The Italian standard NTC-08 [5] follows a strategy that is similar to that of EC8/3 by proposing the same three KLs. For the case of the concrete strength, this standard also introduces a guidance related to what can be regarded as an area with a potentially homogeneous concrete strength. This standard states that the minimum number of material tests must be performed over surface areas smaller than $300 \mathrm{~m}^{2}$. Accordingly, for KL1, one core test must be performed for each type of element, for each storey and for each $300 \mathrm{~m}^{2}$ of construction surface area. For KL2 and KL3, the number of concrete cores that have to be tested is two and three, respectively. To characterize reinforcing steel, the minimum number of tests set by the standard is the same as for concrete but without enforcing the surface area limitation criterion. The values defined by EC8/3 for the CFs of KL1, KL2 and KL3 are also adopted by the Italian standard.

\subsection{Romanian standard $P 100-3$}

The Romanian standard P100-3 [12] follows a material assessment approach similar to that of NTC-08 but sets different minimum values for some of the parameters. The minimum number of concrete core tests that need to be carried out and that P100-3 adopts are referred to a construction area that must not be larger than $1000 \mathrm{~m}^{2}$. Furthermore, these minimum number of tests are now two, four and six for KL1, KL2 and KL3, respectively, for each type of element and for each $1000 \mathrm{~m}^{2}$ of construction surface area. Still, the CF values proposed by EC8/3 are also adopted by P100-3.

\subsection{ASCE 41-13}

The standard ASCE 41-13 [13] defines the material property assessment procedures according to two levels (termed Usual and Comprehensive). Furthermore, it also includes different survey plans to assess the concrete compressive strength and the yield strength of reinforcing steel. For the Usual material assessment level, the evaluation of the concrete strength can be divided in two cases. If the analyst has information about the concrete design strength, at least one core must be extracted from structural components of each different concrete class and the minimum number of cores that need to be tested from the building is three. When the design strength is unknown, at least one core must be extracted from each type of structural component and the minimum number of cores that need to be tested from the building is now six. For reinforcing steel, two cases are also defined for the Usual material assessment level. If design information is available, nominal values of the yield strength can be adopted without the need for testing. If such design data is unavailable, at least two reinforcing steel bars must be extracted from the building for testing. 
Regarding the second level of material assessment defined by ASCE 41-13 (Comprehensive), the minimum number of concrete cores that need to be tested is also divided in cases where design information is available and where it is missing. If the concrete strength specified in the design is known but no additional test data is available, a minimum of three cores must be tested from each storey, each $306 \mathrm{~m}^{3}$ of concrete or each $929 \mathrm{~m}^{2}$ of surface area. When the design concrete strength is unknown and no additional information exists, a minimum of six cores must be tested instead for the same conditions regarding location, surface area and concrete volume. In addition, this standard also specifies that if the $\mathrm{CoV}$ of the concrete core test results is higher than 0.20 , additional tests must be performed until it is lower than or equal to 0.20 . If the additional tests do not reduce the CoV, a knowledge factor of 0.75 must be used to reduce the structural element capacity in the seismic safety assessment (this standard does not reduce the material strength values as the previously analysed standards). To assess the reinforcing steel characteristics, three cases are distinguished for the Comprehensive material assessment. If construction documents are available, at least three reinforcing steel samples must be tested for each type of element. When no information is available about the reinforcing steel grade but the date of the construction is known and the expected reinforcing steel properties are confirmed, at least three samples must be tested for every three storeys and for each type of element. Finally, if the construction date is unknown, at least six steel samples must be tested for every three storeys.

\section{Scope of the proposed $\mathrm{CF}_{\text {mat }}$ safety factor for the mean material strength}

It can be seen from the previous section that existing standards for the seismic safety assessment of existing RC buildings do not provide a unified approach to assess material strength properties and none of the available approaches controls adequately the uncertainty of the in-situ assessment. More specifically, the referred standards involve different approaches to establish the number of tests that need to be performed to estimate the material strength properties and do not address the statistical uncertainty associated to these survey plans. Furthermore, it is likely that different materials may require different assessment approaches given the differences in their expected variability. ASCE 41-13 addresses this aspect by defining different testing plans for the concrete compressive strength and the reinforcing steel yield strength, but does not provide a specific rationale to justify those survey plans. Conversely, since the material property assessment procedure defined by EC8/3 is only disaggregated by storey and by structural element, only the expected construction sequence of a building is likely to be reflected. Since EC8/3 assigns the same $\mathrm{CF}$ values for the concrete strength and the reinforcing steel yield strength, the CF values are disconnected from the expected variability of the materials. Romão et al. [11] addressed this situation by proposing $\mathrm{CF}_{\text {mat }}$ factors for the reduction of the mean material strength according to the expected statistical distribution of the material strength and to the number of tests $(n)$ being performed. The proposed methodology was generic and was used to calibrate specific factors for the mean value of the concrete compressive strength. Nonetheless, in this framework as in others (e.g. see [14]), the uncertainty in the estimates of the material properties depends only on $n$ and does not include any reference to the size and the number of structural elements of the building. However, if the total number of structural elements $N$ is accounted for when defining the survey framework, an explicit control of the sampling uncertainty associated to the number of structural elements where the material strength is not assessed can be achieved.
The methodology proposed herein to derive $\mathrm{CF}_{\text {mat }}$ safety factors extends the original methodology proposed in [11] to include the fact that a building or a region of the building can be divided into $N$ structural elements having an expected homogeneous class of the material strength under assessment [18]. By assuming this finite number of elements, finite population statistics can be considered to define safety factors for the mean value of material strength that account for the uncertainty associated to the survey sampling. When defining $N$, the discrete structure concept is also adopted where each structural member is assumed to be represented by a single strength value which can be obtained from a reliable (destructive) test performed on a material sample from that element. Therefore, for each disaggregated region of $N$ structural elements, the $\mathrm{CF}_{\text {mat }}$ safety factors are defined considering that only a sample of $n$ out of $N$ structural elements are tested and that a prior estimate for the material variability (i.e. the $\mathrm{CoV}$ ) in that region is available.

To derive the referred $\mathrm{CF}_{\text {mat }}$ safety factors, a critical situation in terms of safety also has to be defined. As mentioned before, EC8/3 refers that the estimates of the mean material strength must be divided by the $\mathrm{CF}$ in order to obtain values that have an adequate safety level. Furthermore, the value of $\mathrm{CF}$ is seen to be larger when there is less knowledge about the material. Hence, the underlying critical safety condition justifying the need for the CF reflects a situation where the estimate for the mean material strength overestimates the real value. Therefore, this critical safety condition also needs to be included in the probabilistic quantification of the $\mathrm{CF}_{\text {mat }}$ safety factors proposed herein. Finally, it is noted that the development of the $\mathrm{CF}_{\text {mat }}$ safety factors presented in the following assumes that the statistical distribution of the material strength can be represented by a normal or a lognormal distribution.

\section{Definition of the $\mathrm{CF}_{\text {mat }}$ safety factor for the mean material strength}

\subsection{Definition of $C F_{\text {mat }}$ for the case of a normal distributed strength with known variance}

The proposed $\mathrm{CF}_{\text {mat }}$ safety factor addresses the material strength assessment of a finite population of $N$ members by establishing a confidence interval for the finite population mean. This interval is similar to the common confidence interval for the mean but with the addition of a finite population correction factor which reflects the importance of the relative size of the sample. This correction is based on the fact that when selecting a sample of size $n$ from a finite population of size $N$ that follows a normal distribution, the sample mean $\hat{\bar{x}}_{U}$ follows a normal distribution with a mean equal to the true mean $\bar{x}_{U}$ of the population and a standard deviation $\sigma_{\hat{\bar{x}}_{U}}$ given by [15]:

$\sigma_{\hat{\bar{x}}_{U}}=\sqrt{\mathrm{S}\left(\hat{\bar{x}}_{U}\right)}=\sigma_{U} \cdot \frac{1}{\sqrt{n}} \cdot \sqrt{\frac{\mathrm{N}-n}{N-1}}=\sigma_{U} \cdot \beta_{\mathrm{CoV}}$

where $\sigma_{U}$ is the standard deviation of the population of size $N, \mathrm{~S}\left(\hat{\bar{x}}_{U}\right)$ is the variance of the sampling mean and $\beta_{\mathrm{CoV}}$ is an uncertainty factor that reflects the uncertainty in the estimate of the finite population mean. By standardizing $\hat{\bar{x}}_{U}$, variable $\mathrm{Z}$ is obtained:

$\mathrm{Z}=\frac{\hat{\bar{x}}_{U}-\bar{x}_{U}}{\sigma_{U} \cdot \frac{1}{\sqrt{n}} \cdot \sqrt{\frac{\mathrm{N}-n}{N-1}}}=\frac{\hat{\bar{x}}_{U}-\bar{x}_{U}}{\sigma_{U} \cdot \beta_{\mathrm{CoV}}}$

which follows the standard normal distribution. Using this distribution, the following probability can be obtained: 
$\mathrm{P}\left(-\mathrm{z}_{1-\frac{\alpha}{2}} \leqslant \frac{\hat{\bar{x}}_{U}-\bar{x}_{U}}{\sigma_{U} \cdot \beta_{\mathrm{CoV}}} \leqslant \mathrm{z}_{1-\frac{\alpha}{2}}\right)=1-\alpha$

where $z_{1-\frac{\alpha}{2}}$ is the $\left(1-\frac{\alpha}{2}\right)$ percentage point of the standard normal distribution. Alternatively, if only a one-sided lower bound is needed, the following probability is obtained by modifying Eq. (3):

$\mathrm{P}\left(\frac{\hat{\bar{x}}_{U}-\bar{x}_{U}}{\sigma_{U} \cdot \beta_{\mathrm{CoV}}} \leqslant \mathrm{z}_{1-\alpha}\right)=1-\alpha$

where $z_{1-\alpha}$ is the $(1-\alpha)$ percentage point of the standard normal distribution.

Based on the critical safety condition previously defined where the estimate for the mean $\hat{\bar{x}}_{U}$ is expected to exceed its real value $\bar{x}_{U}$, it is seen that the $\mathrm{CF}_{\text {mat }}$ safety factor must verify the condition:

$\frac{\bar{x}_{U}}{\mathrm{CF}_{\text {mat }}} \leqslant \bar{x}_{U} \leftrightarrow \mathrm{CF}_{\text {mat }} \geqslant \frac{\hat{\bar{x}}_{U}}{\overline{\bar{x}}_{U}}$

Therefore, the minimum value of $\mathrm{CF}_{\text {mat }}$ that still verifies the critical safety condition is:

$\mathrm{CF}_{\text {mat }}=\frac{\hat{\overline{\mathrm{X}}}_{U}}{\overline{\bar{x}}_{U}}$

Combining Eq. (6) with Eq. (4) yields:

$\mathrm{P}\left(\mathrm{CF}_{\text {mat }} \leqslant 1+\mathrm{z}_{1-\alpha} \cdot \mathrm{CoV}_{U} \cdot \beta_{\mathrm{CoV}}\right)=1-\alpha$

where $\mathrm{CoV}_{U}$ is the $\mathrm{CoV}$ of the $N$ material strength values. Eq. (7) states that, for an expected value of the population $\mathrm{CoV}_{U}$ there is a $(1-\alpha)$ probability that $\mathrm{CF}_{\text {mat }} \leqslant 1+\mathrm{z}_{1-\alpha} \cdot \mathrm{CoV}_{U} \cdot \beta_{\mathrm{CoV}}$ if $\bar{x}_{U}=\frac{\hat{\hat{x}}_{U}}{\mathrm{CF}_{\text {mat }}}$. Accordingly, the $(1-\alpha)$ upper confidence bound for $\mathrm{CF}_{\text {mat }}$ is given by:

$\mathrm{CF}_{\text {mat }} \leqslant 1+\mathrm{z}_{1-\alpha} \cdot \mathrm{CoV}_{U} \cdot \beta_{\mathrm{CoV}}$

Since one is interested in establishing a safety factor that will define a limiting value for the mean material strength that is consistent with the critical safety condition previously defined, the maximum value of $\mathrm{CF}_{\text {mat }}$ conforming to the condition set by Eq. (8) must then be adopted:

$\mathrm{CF}_{\text {mat }}=1+\mathrm{z}_{1-\alpha} \cdot \mathrm{CoV}_{U} \cdot \beta_{\mathrm{CoV}}$

Therefore, for a given survey plan (involving $n$ out of $N$ structural elements where the material strength is evaluated), $\mathrm{CF}_{\text {mat }}$ establishes a safety factor for the mean value of the material strength that is compatible with the lower limit of the $(1-\alpha)$ confidence interval that is believed to include the real mean $\bar{x}_{U}$ (Eq. (4)). To quantify $\mathrm{CF}_{\text {mat }}, \mathrm{CoV}_{U}$ needs to be known, but a realistic estimate $\mathrm{CoV} \mid N$ of its expected value can be used instead. This estimate can be defined using values from the literature or survey data from different types of material property tests. Further details regarding the definition of $\operatorname{CoV} \mid N$ for specific materials will be addressed in a later section.

In order to observe the evolution of $\mathrm{CF}_{\text {mat }}$, Fig. 1 presents the evaluation of Eq. (9) for different values of $\operatorname{CoV} \mid N$ (from 0.10 to 0.45 in steps of 0.05 ), for different values of the relative sample size $n / N$ and for different values of the $(1-\alpha)$ confidence level. The minimum value of $\mathrm{CoV} \mid N$ was set to 0.10 since a given material strength will always be affected by multiple sources of uncertainty and it is considered that eliminating all these sources is not feasible for materials used in RC buildings. The maximum value of CoV| $N$ was set to a conservative value of 0.45 that reflects a case with significant heterogeneity in the material properties of a building (e.g. due to a lack of construction or material quality). Four $(1-\alpha)$ confidence levels were also considered to calculate the values for $\mathrm{CF}_{\text {mat }}$ : $0.75,0.85,0.90$ and 0.95 . As discussed in [11], even though there is no evident rationale for the use of these values, they are often referred in the literature as adequate values for ordinary and important structures [16-17]. As expected, the results of Fig. 1 indicate that, irrespective of the selected confidence level, $\mathrm{CF}_{\text {mat }}$ will tend to 1.0 as the ratio $n / N$ also approaches 1.0 . Furthermore, it can also be seen that depending on the selected confidence level and on the expected value of $\operatorname{CoV} \mid N, \mathrm{CF}_{\text {mat }}$ can take values that are higher than the $\mathrm{CF}$ values proposed by the standards previously referred.

\subsection{Definition of $C F_{\text {mat }}$ for the case of a lognormal distributed strength} with known variance

When considering that the material strength follows a lognormal distribution with known variance, an approach similar to that of the normal distribution can be adopted. When considering a random sample of a variable $Y$ extracted from a population having $N$ elements that follow a lognormal distribution with unknown population mean $\bar{y}_{U}$ and known standard deviation $\sigma_{U y}$, the variable $\mathrm{X}=\ln (\mathrm{Y})$ will follow a normal distribution with mean $\bar{x}_{U x}$ and standard deviation $\sigma_{\mathrm{Ux}}$. From the confidence interval defined by Eq. (4), it is known that:

$\hat{\bar{\chi}}_{U x}-\mathrm{z}_{1-\alpha} \cdot \sigma_{U x} \cdot \beta_{\mathrm{CoV}} \leqslant \bar{x}_{U x}$

which, by adding $\sigma_{U x}^{2} / 2$ to both sides and applying the exponential transformation, leads to:

$\mathrm{e}^{\hat{\bar{x}}_{U x}+\frac{\sigma_{U x}^{2}}{2}} \cdot \frac{1}{\mathrm{e}^{\mathrm{z}_{1-\alpha} \cdot \sigma_{U x} \cdot \beta_{\mathrm{COV}}}} \leqslant \mathrm{e}^{\bar{x}_{U x}+\frac{\sigma_{U x}^{2}}{2}}$

where $\mathrm{e}^{\hat{\overline{\mathrm{x}}}_{U x}+\frac{\sigma_{U x}^{2}}{2}}$ represents parameter $\bar{y}_{U}$, i.e. the mean of the lognormal variable Y. Similarly, $\mathrm{e}^{\hat{\mathrm{x}}_{U x}+\frac{\sigma_{U x}^{2}}{2}}$ is the sampling estimate for the mean of variable $Y$, i.e. $\bar{y}_{U}$. Therefore, Eq. (11) can be rewritten as:

$\hat{\overline{\mathrm{y}}}_{U} \cdot \frac{1}{\mathrm{e}^{\mathrm{z}_{1-\alpha} \cdot \sigma_{U x} \cdot \beta_{\operatorname{cov}}}} \leqslant \overline{\mathrm{y}}_{U}$

By the properties of the lognormal distribution, the standard deviation of the associated normal variable $\mathrm{X}$ can be replaced by:

$\sigma_{U x}=\sqrt{\ln \left(\operatorname{CoV}_{U y}^{2}+1\right)}$

where $\mathrm{CoV}_{U y}$ is the CoV of Y. Combining Eq. (12) with Eq. (13) then leads to:

$\hat{\overline{\mathrm{y}}}_{U} \leqslant \overline{\mathrm{y}}_{U} \cdot \mathrm{e}^{\mathrm{z}_{1-\alpha} \cdot \sqrt{\ln \left(\operatorname{CoV}_{U y}^{2}+1\right)} \cdot \beta_{\operatorname{CoV}}}$

Considering that the critical safety condition is now defined as the case where the estimate of the mean $\hat{\bar{y}}_{U}$ exceeds its real value $\overline{\mathrm{y}}_{U}$, the safety factor must verify the condition:

$\frac{\hat{\overline{\mathrm{y}}}_{U}}{\mathrm{CF}_{\text {mat }}} \leqslant \overline{\mathrm{y}}_{U} \leftrightarrow \mathrm{CF}_{\text {mat }} \geqslant \frac{\hat{\overline{\mathrm{y}}}_{U}}{\overline{\overline{\mathrm{y}}}_{U}}$

As before, the minimum value of $\mathrm{CF}_{\text {mat }}$ that still verifies the critical safety condition is:

$\mathrm{CF}_{\text {mat }}=\frac{\hat{\overline{\mathrm{y}}}_{U}}{\overline{\mathrm{y}}_{U}}$

Combining Eq. (16) with Eq. (14) and considering a rationale similar to the one that was assumed for the case where the material strength follows a normal distribution (see Eqs. (7) and (8)) yields:

$\mathrm{CF}_{\text {mat }}=\mathrm{e}^{\mathrm{z}_{1-\alpha} \cdot \sqrt{\ln \left(\mathrm{CoV}_{U y}^{2}+1\right)} \cdot \beta_{\mathrm{CoV}}}$

As in the case of the normally distributed material strength, the parametric definition of $\mathrm{CF}_{\text {mat }}$ depends on the expected value of which is also termed $\operatorname{CoV} \mid N$ herein. In order to observe the 
evolution of $\mathrm{CF}_{\text {mat }}$ for this case, Fig. 2 presents the evaluation of Eq. (17) following the same considerations that were assumed for the case where the material strength follows a normal distribution regarding the range of the selected values for the confidence levels and for $\operatorname{CoV} \mid N$. The results of Fig. 2 can be seen to exhibit an evolution trend similar to that of the results presented in Fig. 1. However, the $\mathrm{CF}_{\text {mat }}$ values are seen to be larger in this case than for the case of the normally distributed material strength, especially for lower values of the ratio $n / N$ and for the higher values of $\operatorname{CoV} \mid N$.

\section{Calibration of a survey framework to assess material strength in $\mathrm{RC}$ buildings}

Since the formulation for the proposed $\mathrm{CF}_{\text {mat }}$ safety factor is similar to the interpretation made by several authors $[10,11]$ regarding the $\mathrm{CF}$ proposed by $\mathrm{EC} 8 / 3$, a survey framework compatible with $\mathrm{EC} 8 / 3$ based on the $\mathrm{CF}_{\text {mat }}$ safety factor was analysed herein. The survey framework includes a direct connection between the $\mathrm{CF}_{\text {mat }}$ safety factors and the KLs and assumes that, for the seismic safety assessment, the mean value of the material strength needs to be factored by $\mathrm{CF}_{\text {mat }}$ to quantify certain parameters, as defined by EC $8 / 3$.

The fundamental change that is introduced by the proposed survey framework refers to the connection between the characteristics of the survey plan and the value of the adopted $\mathrm{CF}_{\text {mat }}$ safety factor. Currently, EC8/3 considers CFs that factor the mean value of the material strength independently of the type of material and that are connected to predefined sampling plans. Instead, a new set of $\mathrm{CF}_{\text {mat }}$ safety factors that depend on a prior estimate of the variability (thus depending on the material) and on the relative number of tested elements $n / N$ is proposed. This proposal overcomes inconsistencies found in the EC8/3 framework that does not account for the total number of structural elements under assessment and does not consider any information about the variability of the material strength to establish the CFs. The proposed survey plans are first discussed in the current section without associating them to a specific material being assessed. The applicability of this general approach to the cases of concrete compressive strength and reinforcing steel yield strength is discussed in Section 6.

\subsection{Alternative definition of the minimum number of tests for each knowledge level $(K L)$}

The proposed survey plans are established for regions of a building where the material properties are believed to be physically homogeneous. An example of these regions refers to the storey differentiation referred in EC8/3 which reflects the expected construction sequence of a building or the disaggregation in groups of storeys proposed by ASCE 41-13 to assess the reinforcing steel properties. Each one of these regions is made of $N$ structural elements and each element is assumed to have a single material strength value.

For each region made of $N$ structural elements, a different relative number of tested elements can be defined that will reflect different KLs about the material properties. Therefore, the proposed procedure establishes minimum values for this relative number of tests $n / N$ for the three KLs of EC8/3 instead of proposing an absolute number of tests that has to be carried out. The proposed survey plans involve the assessment of the material properties in a

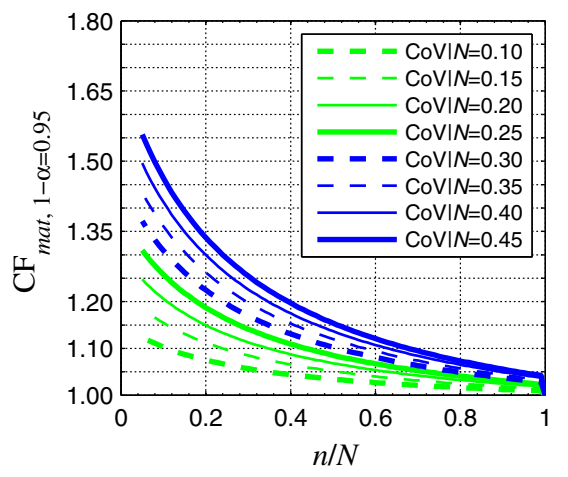

(a)

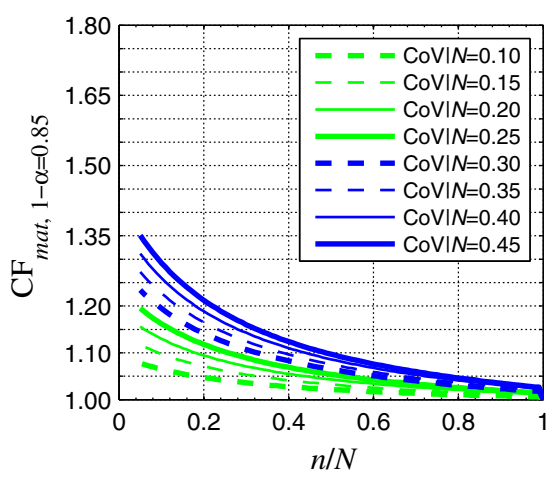

(c)

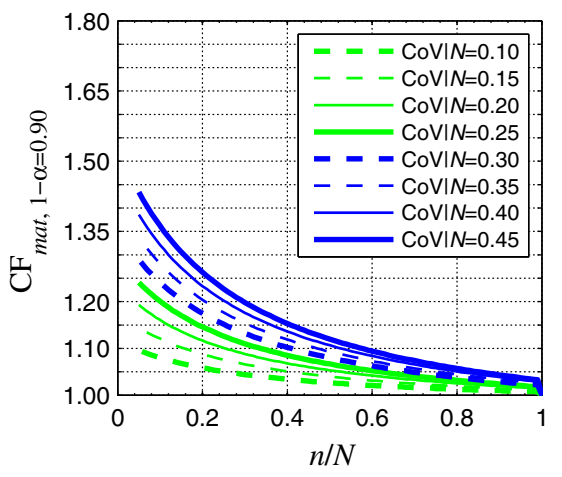

(b)

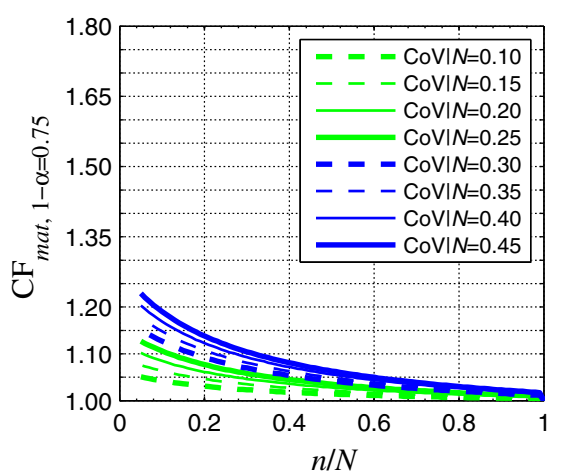

(d)

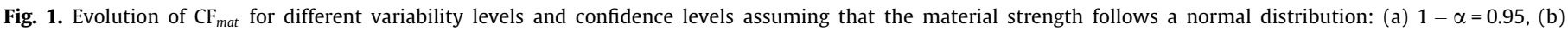
$1-\alpha=0.90$, (c) $1-\alpha=0.85$ and (d) $1-\alpha=0.75$. 


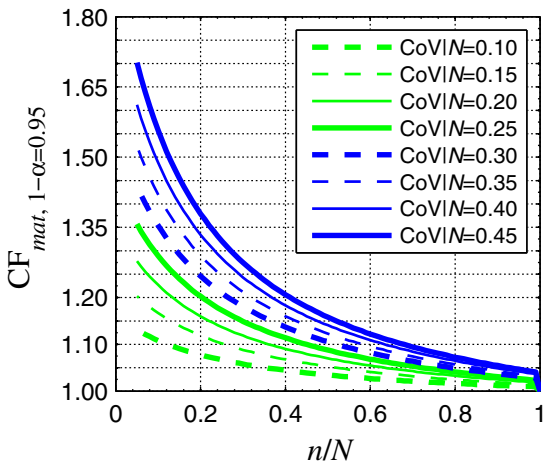

(a)

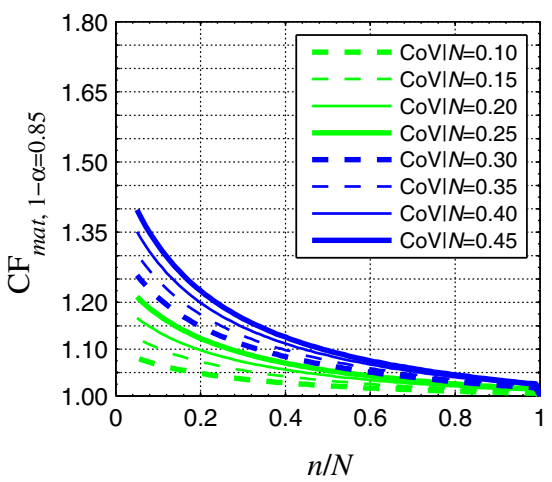

(c)

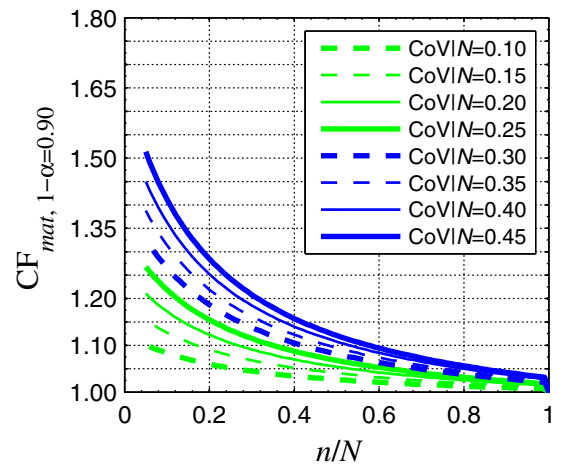

(b)

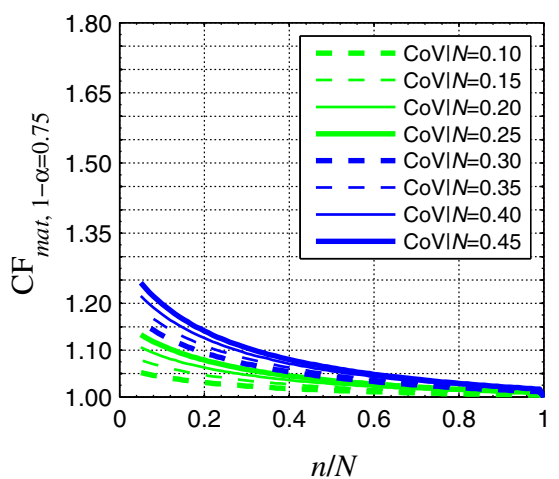

(d)

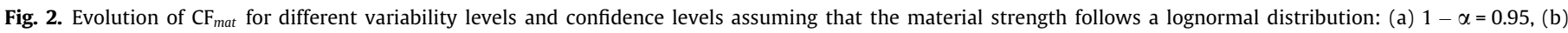
$1-\alpha=0.90$, (c) $1-\alpha=0.85$ and (d) $1-\alpha=0.75$.

minimum number of elements corresponding to $n / N$ ratios equal to $0.1,0.2$ and 0.3 for KL1, KL2 and KL3, respectively. Hence, a higher level of knowledge is obtained when going from KL1 to KL3. For $\mathrm{KL} 3$, the relative sample size of $n / N=0.3$ was established in order to provide a balanced solution between the uncertainty in the estimate for the mean material strength and the structural damage induced to the building during the survey operations [18]. Based on the $n / N$ value set for KL3, values for KL2 and KL1 were defined in order to reflect a reduction in the amount of collected information that would be compatible with the corresponding reduction in the KL. Hence, the suggested sampling plans involving relative sample sizes of $n / N=0.2$ and $n / N=0.1$ reflect a proportional reduction in the number of tests from KL3 to KL2 and from KL2 to KL1, respectively.

To illustrate the proposed survey plans, Fig. 3 presents the evolution of the minimum number $n$ of structural members that need to be tested in regions with a different total number of members (i.e. different values of $N$ ). The values of $n$ presented in Fig. 3 were obtained by rounding up the product between the proposed $n / N$ ratios and each value of $N$ to the nearest following integer. In addition, a complementary condition setting that $n$ must not be lower than two was also enforced for all KLs (two structural members have to be tested to be able to compute the mean value). Results show that for KL1 the number of tests $n$ that is required increases when the value of $N$ increases by ten, e.g. for $11 \leqslant N \leqslant 20, n$ is 2 , for $21 \leqslant N \leqslant 30, n$ is 3 , etc. The relation found for KL2 shows the increase in the number of tests that is required occurs when the value of $N$ increases by five, e.g. for $8 \leqslant N \leqslant 10, n$ is 2 , for $11 \leqslant N \leqslant 15, n$ is 3 , etc. For the case of KL3, the relation found shows the required number of tests increases when the value of $N$ increases by three or four, e.g. for $11 \leqslant N \leqslant 13, n$ is 4 , for $14 \leqslant N \leqslant 16, n$ is 5 , for $17 \leqslant N \leqslant 20, n$ is 6 , etc. These trends were

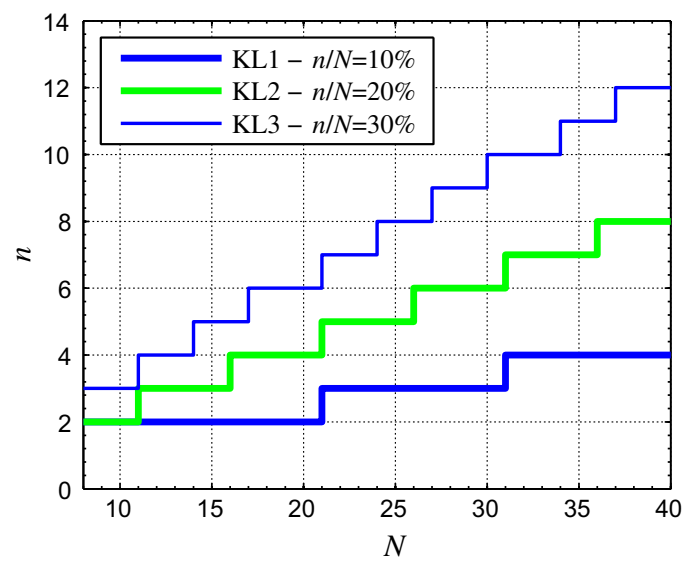

Fig. 3. Variation of the absolute number of tests $n$ for the proposed KLs according to the total number of elements $N$ in the region where the material strength needs to be assessed.

only analysed up to an $N$ value of forty since it was assumed that an $N$ value in this range is representative of the maximum number of structural members of the same type that may be found in an area of $320 \mathrm{~m}^{2}$ (the maximum admissible size of a homogeneous region according to the limit suggested in the Italian standard [5]). If only eight or less structural members are present in the region, a minimum of two tests is always necessary for KL1 and $\mathrm{KL} 2$, and a minimum of three tests is required for KL3. As an example, considering a building storey with twenty structural members (i.e. $N=20$ ), a minimum of two tests is required for KL1, four tests for KL2 and six tests for KL3. 
5.2. Definition of $\mathrm{CF}_{\text {mat }}$ compatible with the proposed knowledge levels and survey plans

To complete the integrated $\mathrm{KL}-\mathrm{CF}_{\text {mat }}$ method proposed herein, a correlation has to be made between the formulation proposed for the $\mathrm{CF}_{\text {mat }}$ safety factors and the survey plans/knowledge levels defined in the previous section. To analyse this correlation, Fig. 4 presents the evolution of the $\mathrm{CF}_{\text {mat }}$ values as a function of increasing values of $\mathrm{CoV} \mid N$, for different (1- $\alpha)$ confidence levels ranging from 0.75 to 0.95 , in steps of 0.05 , and for the three KLs previously defined in terms of $n / N$. The results based on the normal distribution (i.e. Eq. (10)) are $\mathrm{CF}_{\text {mat }}$ presented in Fig. 4a (KL1), Fig. 4b (KL2) and Fig. 4c (KL3), while those based on the lognormal distribution (i.e. Eq. (18)) are presented in Fig. 4d (KL1), Fig. 4e (KL2) and Fig. 4f (KL3).

A global analysis of the results of Fig. 4 indicates that, for each $\mathrm{KL}$ and for all the selected confidence levels, the values of $\mathrm{CF}_{\text {mat }}$ are larger when assuming a lognormal distribution. These differences, and the fact that it represents a more conservative approach, indicate that this model is more adequate to define the values of $\mathrm{CF}_{\text {mat }}$ within a safety assessment perspective where no information about the distribution shape is available. After setting this condition, it is necessary to decide which confidence level should be assigned to each KL. Little guidance can be found with respect to the selection of an adequate confidence level to establish material strength values. Still, some rationale seems to exist regarding the bounds for possible values of the confidence level. As referred in [11], a minimum confidence level of 0.75 is generally considered in the context of structural assessment. On the other hand, it is common to find the value of 0.95 being suggested as a maximum value for all practical purposes. As can be seen from Fig. 4, all KLs exhibit significant differences between the values obtained for the 0.95 and 0.75 confidence levels. On the other hand, the results obtained for the 0.85 and the 0.80 confidence levels are very similar. Also, the results obtained for the 0.85 confidence level are closer to those obtained for the 0.75 confidence level than to those of the 0.95 confidence level.

Given these results, the maximum confidence level analysed, i.e. 0.95 , could be recommended in order to be more confident that the true unknown mean will not be lower than the estimate corrected by the $\mathrm{CF}_{\text {mat }}$. Figure 5a presents the three curves representing the interconnection between the KL and assuming a constant 0.95 confidence level for all KLs. However, since the three KLs are associated with three different amounts of available data, the case where the confidence level associated to the $\mathrm{CF}_{\text {mat }}$ of each $\mathrm{KL}$ could be different was also analysed. Therefore, instead of selecting the same confidence level for all the KLs, one may alternatively require a higher confidence level (i.e. a larger confidence interval) for KL1 since there is less information for that KL. For the remaining KLs, lower confidence levels (i.e. with smaller confidence intervals) may, therefore, be progressively established. This fact can be analysed bearing in mind the reduction of the sampling uncertainty about the mean that is obtained when $n / N$ increases. Hence, if one assumes a maximum confidence level of 0.95 for the case where $n / N$ is lower (i.e. $\mathrm{KL} 1$ ), the minimum confidence level of 0.75 can be associated to the case where $n / N$ is larger (KL3). An intermediate confidence level may then be established for KL2. Since the reduction of is approximately $50 \%$ from a confidence level of 0.90 to a confidence level of 0.75 , the value of 0.90 was assumed for the intermediate level of knowledge (KL2). Figure 5b presents the three curves representing the interconnection between the $\mathrm{KL}$ and $\mathrm{CF}_{\text {mat }}$ assuming different confidence levels for each KL. As expected, this approach leads to lower safety factors for KL2 and KL3 than the one where a confidence level of 0.95 is assumed (Fig. $5 a$ ).

\subsection{Comparison between the EC8/3 CF values and the proposed values}

The $\mathrm{CF}_{\text {mat }}$ safety factor established for the three KLs can be compared with the CF values proposed by other standards. As referred before, EC8/3 and the Italian code propose a similar approach

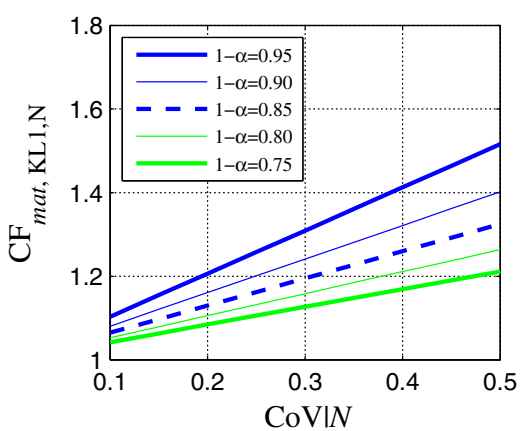

(a)

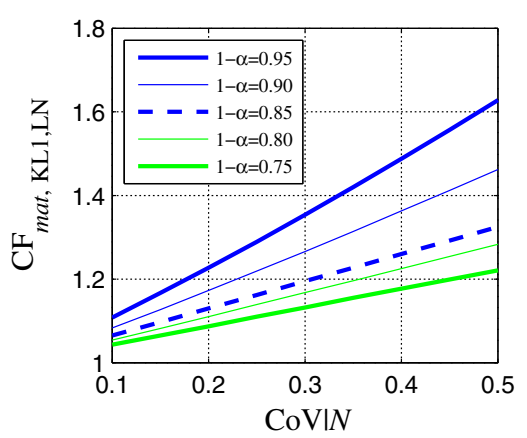

(d)

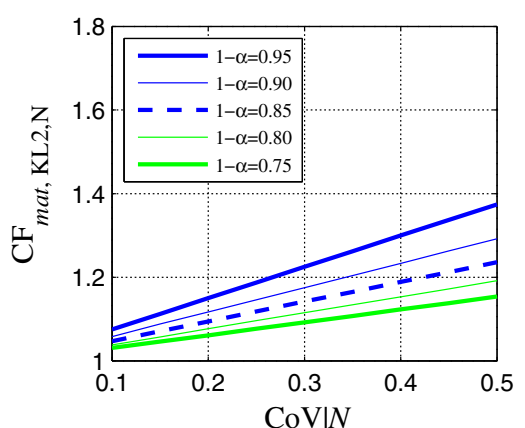

(b)

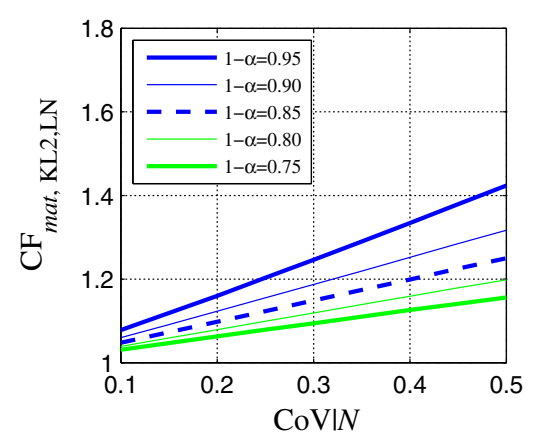

(e)

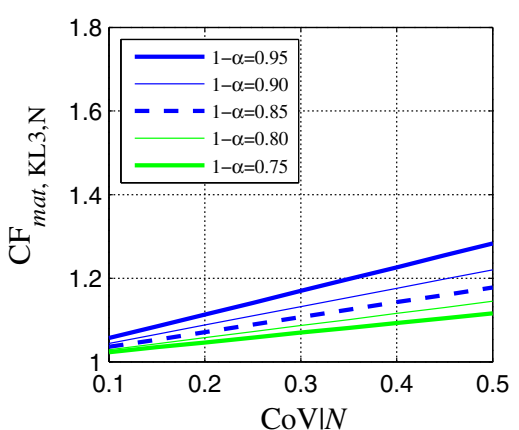

(c)

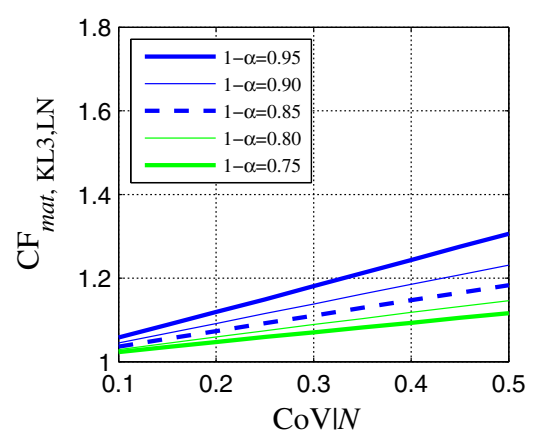

(f)

Fig. 4. Evolution of $\mathrm{CF}_{\text {mat }}$ for different KLs assuming a normal distribution (KL1, (a); KL2, (b); KL3, (c)) and a lognormal distribution (KL1, (d); KL2, (e); KL3, (f)). 


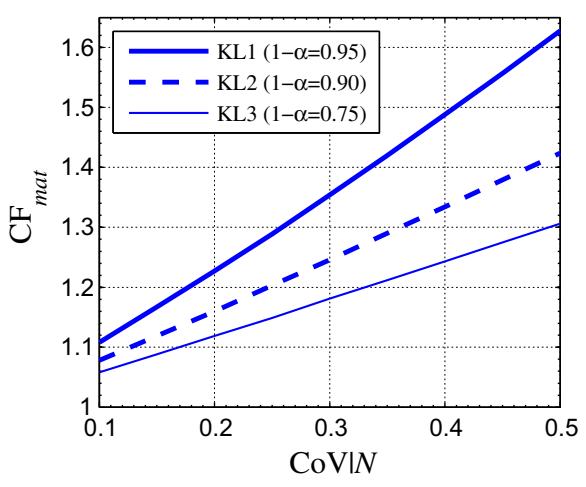

(a)

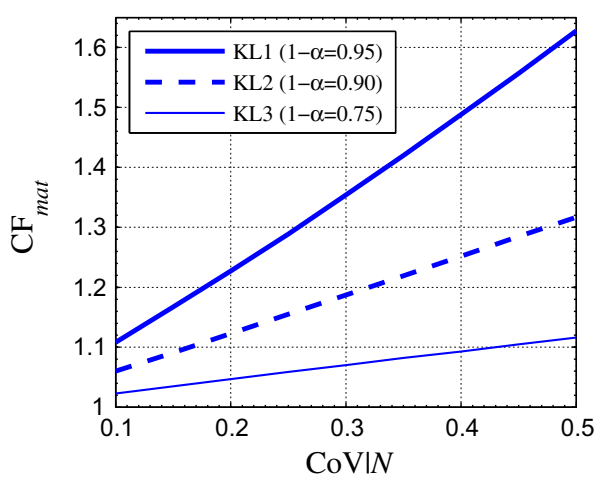

(b)

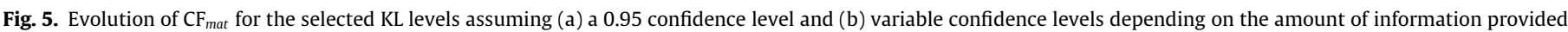
by the survey plan compatible with the KL.

regarding the survey operations that are needed to assess material strength. Still, it must be noted that the CF value proposed by these standards for $\mathrm{KL} 3(\mathrm{CF}=1.0)$ is unrealistic unless the material strength is assessed in all the structural members. Given the $\mathrm{CF}$ values these standards propose for KL2 and KL1, 1.20 and 1.35, respectively, a reference value of 1.10 is proposed for the CF of $\mathrm{KL} 3$ for the purpose of the following analysis. To analyse the two approaches, Fig. 6 shows the comparison of the (fixed) $\mathrm{CF}-\mathrm{KL} 1_{\mathrm{EC} 8 / 3}, \mathrm{CF}-\mathrm{KL} 2_{\mathrm{EC} 8 / 3}$ and $\mathrm{CF}-\mathrm{KL} 3_{\mathrm{EC} 8 / 3}$ factors associated to the KLs according to EC8/3, and the (variable) safety factors considering the different confidence levels previously assigned.

By comparing the $\mathrm{CF}$ values and the evolution of the proposed safety factors, it can be seen that for both approaches to be compatible, the admissible variability of the material property (i.e. $\mathrm{CoV} \mid N)$ must be limited. By analysing the range of CoVs for which $\mathrm{CF}-\mathrm{KL} 1_{\mathrm{EC} / 3}$ and $\mathrm{CF}-\mathrm{KL} 2_{\mathrm{EC} 8 / 3}$ cross their corresponding curve (CF$\mathrm{KL} 3_{\mathrm{EC} / 3}$ was left out since 1.10 is not the true value proposed by the standards), it can be seen that $\operatorname{CoV} \mid N$ should be limited to a value around 0.30 . For $\mathrm{KL} 1$, a $\mathrm{CoV} \mid \mathrm{N}$ of 0.30 leads to a $\mathrm{CF}_{\text {mat }}$ value of 1.34 while, for KL2, a value of 1.18 is obtained. For KL3, the $\mathrm{CF}_{\text {mat }}$ value corresponding to a $\mathrm{CoV} \mid \mathrm{N}$ of 0.30 is 1.07 .

Based on this analysis, the CF values proposed by EC8/3 and the Italian standard for KL1 and KL2 can only be found to be acceptable for the purpose of defining a safe value of the mean material strength as long as $\operatorname{CoV} \mid N$ is lower than 0.30. For the case of KL3, the proposed approach based on $\mathrm{CF}_{\text {mat }}$ leads to a more statistically

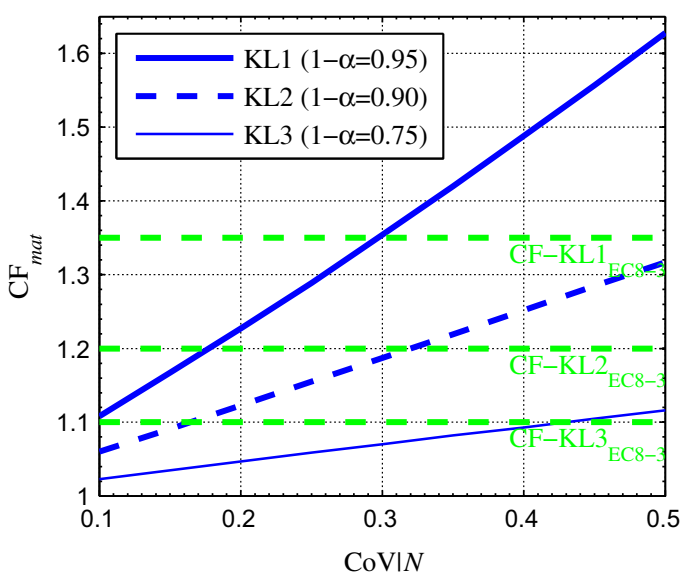

Fig. 6. Evolution of $\mathrm{CF}_{\text {mat }}$ for the selected confidence levels of each $\mathrm{KL}$ and comparison with the $\mathrm{CFs}$ proposed by $\mathrm{EC} 8 / 3$ and the Italian standard considering variable confidence levels. sound proposal since the $\mathrm{CF}$ value of 1.0 proposed by the EC8/3 and the Italian standard is unrealistic. Nonetheless, the standard-based $\mathrm{CF}$ values will lead to overconservative values of the mean material strength when $\mathrm{CoV} \mid N$ is significantly lower than the referred limit of 0.30 . Therefore, an approach defining a $\mathrm{CF}_{\text {mat }}$ that varies according to the level of material strength variability that is found (or expected) during the assessment is seen to be more flexible and useful. As such, this approach enables the definition of different $\mathrm{CF}_{\text {mat }}$ values for different types of materials.

\section{Defining $\mathrm{CF}_{\text {mat }}$ safety factors for concrete and reinforcing steel}

EC8/3 defines KLs and CFs for the assessment of material properties without distinguishing the type of material. Therefore, according to the European code, the same number of structural elements should be tested in each storey of a RC building for the quantification of the concrete compressive strength and the reinforcing steel yield strength. On the contrary, since the proposed integrated $\mathrm{KL}-\mathrm{CF}_{\text {mat }}$ method depends on an estimate of the material strength variability $\operatorname{CoV} \mid N$, different strategies and different $\mathrm{CF}_{\text {mat }}$ values can be defined for these two different materials.

For the case of the concrete compressive strength, a $\mathrm{CF}_{\text {mat }}$ termed $\mathrm{CF}_{\text {conc }}$ can be defined which will depend directly on the estimate of the dispersion of the $N$ concrete strength values $\operatorname{CoV}_{f c} \mid N$. As shown in previous studies [18-19], estimating the $\operatorname{CoV}_{f c} \mid N$ using a small sample of results from concrete core tests may lead to estimates that do not reflect the real variability of the concrete strength. This fact is even more relevant due to the high value of the concrete strength $\operatorname{CoV}_{f c} \mid N$ that is usually found in existing buildings [20-23], often exceeding a value of 0.20 [24]. A methodology improving the accuracy of the estimate of $\operatorname{CoV}_{f c} \mid N$ by using rebound hammer tests was proposed in [18]. Using results of the rebound hammer test, i.e. the rebound numbers (RNs), carried out in a minimum number of $n / N=0.30$ elements in a region, the methodology determines their variability, $\operatorname{CoV}_{R N} \mid n$, and converts it into an equivalent value of $\operatorname{CoV}_{f c} \mid N$ using an empirical model. Details on the adequacy of this methodology to estimate the concrete strength variability can be found in [18]. Alternatively, a conservative approach can be adopted to establish generic values for $\mathrm{CF}_{\text {conc. }}$ Given the range of values reported in the literature (e.g. see [20-24]), a $\mathrm{CoV}_{f c} \mid N$ of 0.30 can be considered to be a conservative estimate of the concrete strength variability. According to Fig. 6 and to the assumptions it involves (see Sections 5.2 and 5.3), the $\mathrm{CF}_{\text {conc }}$ values that are obtained by considering a $\mathrm{CoV}_{f c} \mid N$ of 0.30 are $1.34,1.18$ and 1.07 , for $\mathrm{KL1}$, KL2 and KL3, respectively. However, for simplicity, it is suggested to round these values and 


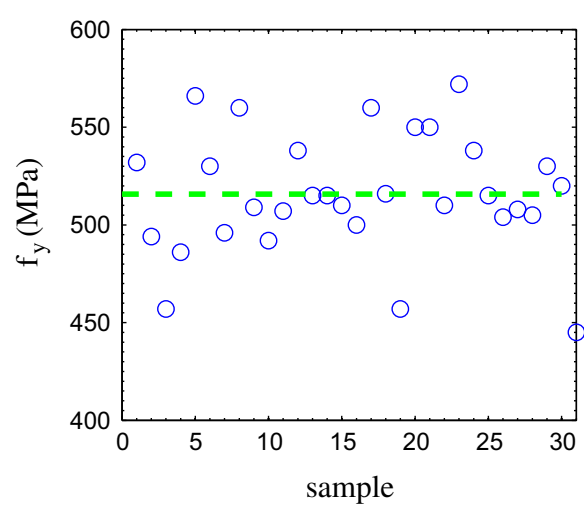

(a)

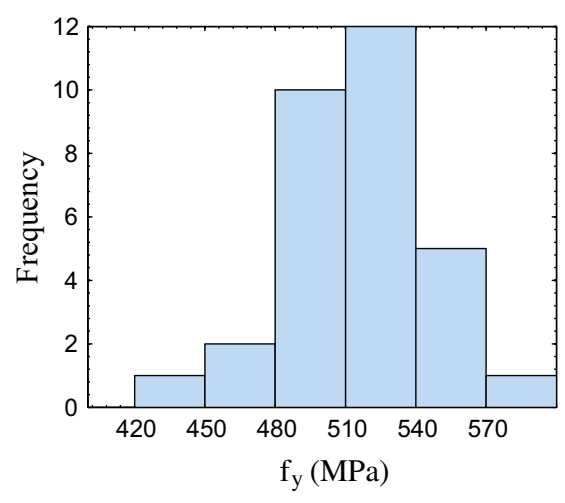

(b)

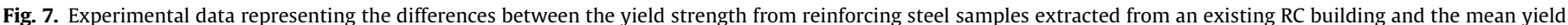
strength (dashed line) (a) and corresponding histogram of the experimental data (b).

define the $\mathrm{CF}_{\text {conc }}$ values as $1.35,1.20$ and 1.10 for KL1, KL2 and KL3, respectively.

For the case of reinforcing steel, common values for the CoV|N of the rebar yield strength are generally less than 0.10 . Several studies characterizing the steel yield strength can be found in the literature to support this level of variability. For example, experimental results from [25] showed that for reinforcing steel bars with a nominal strength of $280 \mathrm{MPa}$, a $\mathrm{CoV}$ of 0.107 was found, while for a nominal strength of $410 \mathrm{MPa}$, the variability decreased to 0.093. Moreover, experimental results from [26] showed that for reinforcing steel bars of the European class S400, a CoV of 0.047 was found, while for reinforcing steel bars of the European class S500, a CoV of 0.052 was obtained. The analysis of experimental results obtained by the authors from tensile tests of reinforcing steel bars extracted from an existing building led to the yield strength variations shown in Fig. 7. The tests were performed in reinforcing bars with $\varnothing 16(\mathrm{~mm})$ which were expected to conform with the European Class S500. A mean yield strength of $515 \mathrm{MPa}$ was found for the 31 tested samples, with a CoV of 0.06. Although these values can be assumed as a reference, a conservative estimate for $\mathrm{CoV} \mid \mathrm{N}$ with a value of 0.10 might be more adequate for existing structures due to potential alterations in the characteristics of the reinforcing bars.

Considering a $\operatorname{CoV} \mid N$ with a value of 0.10 for the reinforcing steel yield strength, $\mathrm{CF}_{\text {mat }}$ (in this case termed $\mathrm{CF}_{\mathrm{rs}}$ ) values of $1.10,1.06$ and 1.02, for KL1, KL2 and KL3, respectively, are found to be compatible with Fig. 6 and the assumptions it involves. However, since repairing the damage caused by extracting reinforcing steel bar samples from a RC structure for testing is expected to be more expensive than repairing the holes left after extracting concrete cores (additional concrete needs to be removed to provide adequate lap splicing and formwork will also be needed), there are some practical advantages in revising the values of $n / N$ that are

Table 1

Number of tests to be performed at each region made of $\mathrm{N}$ structural members.

\begin{tabular}{llllll}
\hline $\begin{array}{l}\text { Knowledge } \\
\text { level }\end{array}$ & $\begin{array}{l}\text { Concrete } \\
\mathrm{NDTs}^{\mathrm{a}} \\
(n / N)\end{array}$ & $\begin{array}{l}\text { Concrete } \\
\text { core tests } \\
(n / N)\end{array}$ & $\mathrm{CF}_{\text {conc }}{ }^{\mathrm{b}}$ & $\begin{array}{l}\text { Reinforcing } \\
\text { steel tensile } \\
\text { tests }(n / N)\end{array}$ & $\mathrm{CF}_{\mathrm{rs}}{ }^{\mathrm{c}}$ \\
\hline (KL1) Limited & 0.30 & 0.10 & 1.35 & 0.05 & 1.15 \\
(KL2) Comprehensive & 0.30 & 0.20 & 1.20 & 0.05 & 1.10 \\
(KL3) Full & 0.30 & 0.30 & 1.10 & 0.05 & 1.05
\end{tabular}

\footnotetext{
a Suggested values assume that NDTs are rebound hammer tests but other NDTs can also be used.

b Assuming that $\mathrm{CoV} \mid \mathrm{N}$ of the concrete compressive strength is lower than 0.30 .

c Assuming that $\mathrm{CoV} \mid \mathrm{N}$ of the steel yield strength is lower than 0.10 .
}

proposed for the different KLs. Therefore, instead of considering $n / N$ values of $0.10,0.20$ and 0.30 for KL1, KL2 and KL3, respectively, to characterize the reinforcing steel yield strength, a constant $n / N$ value of 0.05 is now suggested. By analysing the results of Fig. 2 for a $\mathrm{CoV} \mid N$ of 0.10 , it can be seen that the $\mathrm{CF}_{\text {mat }}$ values corresponding to $n / N=0.05$ are $1.13,1.10$ and 1.05 , for KL1, KL2 and KL3, respectively. These $\mathrm{CF}_{\text {mat }}$ values can be seen to be slightly larger than those obtained for the previous $n / N$ values and suggest that $\mathrm{CF}_{\mathrm{rs}}$ values of $1.15,1.10$ and 1.05 can be proposed for KL1, KL2 and KL3, respectively, to characterize the mean yield strength of reinforcing steel involving the assessment of only $n / N=0.05$ structural elements for all the KLs. It is noted that for lower values of $N$, the $n / N=0.05$ condition can lead to the need of only one test to estimate the reinforcing steel yield strength in a region. Still, conceptually, a minimum of two tests is required to compute an estimate for the mean.

Based on the $\mathrm{CF}_{\text {conc }}$ and $\mathrm{CF}_{\mathrm{rs}}$ values that were defined for the several KLs, Table 1 summarizes a proposal for a survey framework that can be used in a standard-based approach to assess the concrete compressive strength and the steel yield strength in existing RC buildings. This proposal defines sampling plans for concrete NDTs, concrete core compression tests and reinforcing steel sample tensile tests by specifying the minimum $n / N$ number of tests that needs to be performed at each region made of $N$ structural members (e.g. a storey) and for each type of structural element.

\section{Conclusions}

The present study proposed an adaptive probability-based framework defining test sampling plans for existing RC buildings and new $\mathrm{CF}_{\text {mat }}$ material safety factors leading to mean material strength values that are on the "safe side". The development of the framework is also based on two essential concepts: (1) a building can be divided into one or more regions, where each region has $N$ structural elements and is expected to exhibit a homogeneous class of the material strength under assessment; (2) each structural element from a given region is defined by a single value of the material strength under assessment. By assuming this finite number of elements and of material strength values in each region, the proposed framework uses finite population statistics to define $\mathrm{CF}_{\text {mat }}$ safety factors that consider the uncertainty associated to the number of tested structural elements in a region and the inherent variability of the material strength under analysis. Analytical expressions were defined for the $\mathrm{CF}_{\text {mat }}$ safety factors for the case where the material property is assumed to follow a normal distribution and for the case where it is assumed to follow a lognormal 
distribution. These expressions rely on the possibility of quantifying the expected material strength variability and possible approaches were discussed to estimate this variability.

The proposed framework was developed in order to be compatible with seismic safety assessment procedures defined by current standards such as EC8/3, namely by also considering the concept of $\mathrm{KL}$ and by defining test sampling plans and $\mathrm{CF}_{\text {mat }}$ safety factors in agreement with the KLs established by these standards. For these $\mathrm{KLs}$, the definition of the $\mathrm{CF}_{\text {mat }}$ safety factors was analysed for different values of the expected material variability and for different confidence levels. Based on these analyses, confidence levels of 0.95, 0.90 and 0.75 were proposed for KL1, KL2 and KL3, respectively, to establish a connection between the values of $\mathrm{CF}_{\text {mat }}$ and the KLs. A comparison between the proposed $\mathrm{CF}_{\text {mat }}$ safety factors and the $\mathrm{CF}$ values defined by EC8/3 showed that the latter can only provide conservative results (i.e. on the safe side) if the $\mathrm{CoV}$ of the material in the region being assessed is below 0.30 .

Specific $\mathrm{CF}_{\text {mat }}$ safety factors were then defined for the concrete compressive strength and for the reinforcing steel yield strength, termed $\mathrm{CF}_{\text {conc }}$ and $\mathrm{CF}_{\mathrm{rs}}$, respectively that account for their different variability. Finally, specific values of the minimum number of destructive and non-destructive tests that have to be performed in a region of a RC building to characterize these material strength properties were also established. The format of the proposed test sampling plans and of the $\mathrm{CF}_{\text {conc }}$ and $\mathrm{CF}_{\mathrm{rs}}$ safety factors is suitable for integration in standard-based procedures such as those of EC8/3 and overcomes some of their previously highlighted limitations.

\section{References}

[1] CEN, Eurocode 8: design of structures for earthquake resistance. Part 3: Assessment and retrofitting of buildings. Brussels, 2005.

[2] NZSEE, Assessment and Improvement of the Structural Performance of Buildings in Earthquake. Recommendations of a NZSEE Study Group on Earthquake Risk Buildings, New Zealand Society for Earthquake Engineering, New Zealand, 2006.

[3] ASCE, Seismic Evaluation of Existing Buildings (ASCE/SEI 31-03), American Society of Civil Engineers, Reston, Virginia, USA, 2004.

[4] ASCE, Seismic Rehabilitation of Buildings (ASCE/SEI 41-06), American Society of Civil Engineers, Reston, Virginia, USA, 2007.

[5] Circolare 2 febbraio 2009. n. 617 Approvata dal consiglio superiore dei Lavori Pubblici. Istruzioni per l'applicazione delle "Nuove norme tecniche per le costruzioni" di cui al decreto ministeriale 14 gennaio, 2008-2009 [in Italian].

[6] ATC, Quantification of Building System Performance and Response Parameters, Applied Technology Council, Redwood City, California, USA, 2009.
[7] L. Elefante, Dealing with uncertainties in the assessment of existing RC buildings PhD dissertation, Università degli Studi di Napoli Federico II, Naples, Italy, 2009.

[8] P. Franchin, P. Pinto, P. Rajeev, Confidence factor?, J Earthq. Eng. 14 (7) (2010) 989-1007.

[9] M. Rota, A. Penna, G. Magenes, A framework for the seismic assessment of masonry buildings taking into account different sources of uncertainty, J. Earthq. Eng. Struct. Dyn. 43 (7) (2014) 1045-1066.

[10] G Monti, S. Alessandri, Application of Bayesian techniques to material strength evaluation and calibration of confidence factors, in: E. Cosenza (Ed.), Eurocode 8 Perspectives From the Italian Standpoint Workshop, 67-77, Doppiavoce, Naples, Italy, 2009.

[11] X. Romão, R. Gonçalves, A. Costa, R. Delgado, Evaluation of the EC8-3 confidence factors for the characterization of concrete strength in existing structures, Mater. Struct. 45 (11) (2012) 1737-1758.

[12] MDRT, Seismic Design Code. Part III. Seismic assessment of existing buildings, Monitorul Official No. 647 bis (P 100-3/2008), 2009.

[13] ASCE, Seismic Evaluation and Retrofit of Existing Buildings (ASCE/SEI 41-13) American Society of Civil Engineers, Reston, Virginia, USA, 2014.

[14] Y. Kog, Sample size for determining in situ strength of concrete in structures, Mag. Concr. Res. 64 (11) (2012) 1045-1048.

[15] P. Levy, J. Lemeshow, Sampling of Populations: Methods and Applications, 4th ed., Wiley, New York, 2008.

[16] K. Hindo, W. Bergstrom, Statistical evaluation of the in-place compressive strength of concrete, Concr. Int. 7 (2) (1985) 44-48.

[17] W.F. Wong, S.P. Chiew, N.Y. Ho, Evaluation of in situ test data from existing concrete structures, in: R.K. Dhir, M.R. Jones (Eds.), Proceedings of the Symposium Concrete 2000: Economic and Durable Construction Through Excellence, vol. vol 2, E \& FN Spon, London, 1993.

[18] N. Pereira, X. Romão, Assessment of the concrete strength in existing buildings using a finite population approach, Constr. Build. Mater. 110 (2016) 106-116.

[19] A. Fiore, F. Porco, G. Uva, M. Mezzina, On the dispersion of data collected by in situ diagnostic of the existing concrete, Constr. Build. Mater. 47 (2013) 208217.

[20] Y. Shimizu, M. Hirosawa, J. Zhou, Statistical Analysis of Concrete Strength in Existing Reinforced Concrete Buildings, in: Proceedings of 12th World Conference on Earthquake Engineering, Japan, 2000.

[21] MT Cristofaro, A D’Ambrisi, M De Stefano, R Pucinotti, M Tanganelli, Studio sulla Dispersione dei Valori di Resistenza a Compressione del Calcestruzzo di Edifici Esistenti, Il Giornale delle Prove non Distruttive Monitoraggio e Diagnostica (2012) 2 (in Italian)

[22] A. Masi, A. Digrisolo, G. Santarsiero, Concrete strength variability in Italian RC buildings: analysis of a large database of core tests, Appl. Mech. Mater. 597 (2014) 283-290.

[23] A. Masi, L. Chiauzzi, An experimental study on the within-member variability of in situ concrete strength in RC building structures, Constr. Build. Mater. 47 (2013) 951-961.

[24] A. Masi, M. Vona, Estimation of the in-situ concrete strength: provisions of the European and Italian seismic codes and possible improvements, in: E. Cosenza (Ed.), Eurocode 8 Perspectives from the Italian Standpoint Workshop, 67-77, Doppiavoce, Naples, Italy, 2009.

[25] S. Mirza, J.G. MacGregor, Variability of the mechanical properties of reinforcing bars, J. Struct. Div 105 (1979) 921-937.

[26] M. Pipa, Ductilidade de elementos de betão armado sujeitos a acçoes cíclicas: Influencia das caracteristicas mecâncias das armaduras PhD dissertation, Universidade Técnica de Lisboa, Lisbon, Portugal, 1993 (in Portuguese). 